\title{
REVISÃO DOS TRATAMENTOS QUÍMICOS DA FIBRA NATURAL PARA MISTURA COM POLIOLEFINAS
}

\author{
Sandra Regina Albinante, Élen Beatriz Acordi Vasques Pacheco* e Leila Lea Yuan Visconte \\ Instituto de Macromoléculas Professora Eloisa Mano, Universidade Federal do Rio de Janeiro, CP 68525, 21941-972 Rio de \\ Janeiro - RJ, Brasil
}

Recebido em 5/1/12; aceito em 13/7/12; publicado na web em 29/11/12

\begin{abstract}
A REVIEW ON CHEMICAL TREATMENT OF NATURAL FIBER FOR MIXING WITH POLYOLEFINS. The use of natural fibers as reinforcement in polymer composites has been a focus of interest. However, these composites exhibit lower mechanical properties than those of pure polymers because of the low interfacial interactions between the hydrophobic polymer matrix and the hydrophilic fiber. To overcome this problem, different chemical treatments applied to the fibers have been reported. One of the most used treatments is mercerization, which can improve adhesion between the fiber and polymeric matrix. Another chemical treatment involves the use of acids (stearic and oleic acids). The chemically treated fibers used in composite materials showed improved mechanical properties.
\end{abstract}

Keywords: natural fibers; chemical treatment; composites.

\section{INTRODUÇÃO}

Os resíduos da agricultura são constituídos principalmente de fibras vegetais, cujo componente químico principal é a celulose. Esses resíduos naturais podem ser facilmente misturados a materiais termoplásticos para a produção de compósitos. ${ }^{1-3}$

As fibras vegetais oferecerem vantagens como baixo custo, baixa densidade, excelente resistência a solventes e à temperatura. Além disso, são atóxicas e não abrasivas, sendo facilmente modificadas por agentes químicos. ${ }^{2}$

Essas características tornam as fibras vegetais materiais tecnologicamente interessantes em diversas aplicações, como em carpetes, vasos, cordas, telhas, estofados de automóveis, colchões, entre outras. ${ }^{1-6}$ Entre os exemplos de fibras naturais brasileiras estão as fibras de coco, da bananeira, da palma, de curauá, de sisal, de juta, do bagaço de cana-de-açúcar, das palhas de arroz e trigo, da piaçava, do algodão.

Apesar das excelentes propriedades e vantagens quanto à utilização, as fibras naturais apresentam também algumas desvantagens quando utilizadas como cargas em compósitos poliméricos, como desempenho mecânico inadequado, baixa termoplasticidade e temperatura de processamento, alta absorção de umidade e incompatibilidade com termoplásticos mais comuns. ${ }^{7-9}$

A maior parte dos problemas relacionados às fibras naturais se deve aos grupamentos hidrofílicos presentes em sua estrutura química. Esses grupos conferem às fibras naturais uma característica polar, enquanto os polímeros olefínicos são apolares. Misturas entre diferentes materiais apresentam uma interação interfacial muito fraca, o que resulta em produtos finais com propriedades mecânicas inferiores às dos polímeros puros. Para se obter uma mistura polimérica uniforme e com boas propriedades, é necessário aumentar a interação entre os componentes, o que pode ser conseguido submetendo a fibra, ou o polímero, a um tratamento químico., ${ }^{4-13}$

Tratamentos químicos na fibra natural vêm sendo amplamente pesquisados e utilizados. Entre eles, podem-se citar a mercerização, o tratamento com ácidos, com grupos silano, acetil, isocianato, permanganato e peróxido. Esses tratamentos atuam melhorando a interface entre a fibra e a matriz. ${ }^{10,14-16}$

O objetivo deste artigo foi mostrar as diferentes formas de tratamento químico em fibras naturais e os resultados sobre as propriedades dos compósitos de poliolefinas com essas fibras modificadas.

\section{Composição química das fibras naturais}

Fibras naturais são materiais de origem animal, mineral ou vegetal. ${ }^{17}$ As fibras são constituídas de células alongadas entrelaçadas e o entrelaçamento delas é, em parte, responsável por algumas propriedades e características como densidade, tensão, módulo e alongamento na ruptura, como mostrado na Figura 1.10,18

As fibras naturais têm cinco componentes básicos: celulose, hemicelulose, pectina, lignina e extrativos (gorduras, proteínas e sais inorgânicos). A celulose (40 a 90\% em massa da fibra) é responsável pela resistência das fibras, devido ao seu alto grau de polimerização e orientação molecular. É um polímero linear cristalino formado por unidades $\beta$-D-glicopironases unidas por ligações glicosídicas. A hemicelulose (1 a $30 \%$ em massa) é uma variedade de moléculas complexas, amorfas e de unidades $\beta$-D-xilose, $\beta$-D-manose, $\beta$-D-glicose, $\alpha$-L-arabinose e ácido $\beta$-D-glicurônico. Essas unidades são formadas por cadeias de carbono com um grupo hidroxila, exceto os que podem estar na forma de carbonila ou em ligação hemiacetal. A lignina, segundo maior componente em massa (1 a 35\% em massa), é uma macromolécula formada por um sistema aromático, reticulado, com elevada massa molar, amorfo e com unidades de fenilpropano (Figura 2). ${ }^{18,19}$

O conhecimento da estrutura interna das fibras lignocelulósicas e da sua composição química é importante para a compreensão de sua influência nas características do compósito e, também, na escolha dos tratamentos químicos. As propriedades químicas e físicas das fibras estão associadas às diferentes proporções de seus cinco componentes, que variam conforme o tipo da fibra (Figura 3). ${ }^{18,19}$

\section{Tratamentos das fibras}

Para aumentar a adesão interfacial entre as fibras naturais e a poliolefina, várias estratégias têm sido propostas, dentre elas os tratamentos químicos. Os efeitos destes tratamentos variam de acordo com a composição da fibra, principalmente em relação à proporção de celulose.

\section{Tratamentos químicos}

Os tratamentos químicos são aqueles em que se emprega algum 
a)

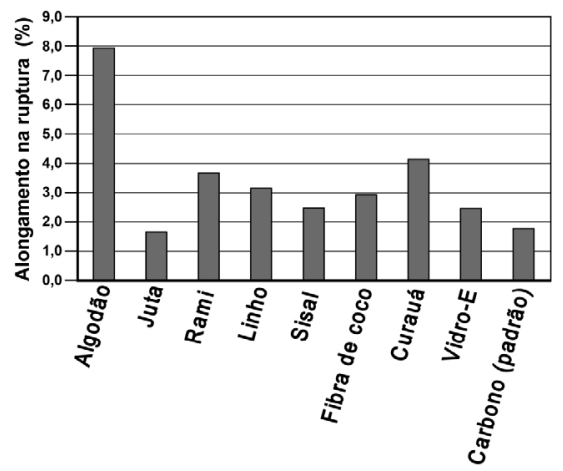

b)

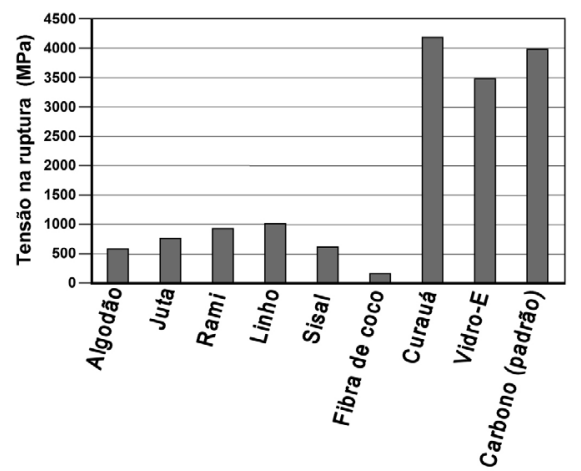

c)

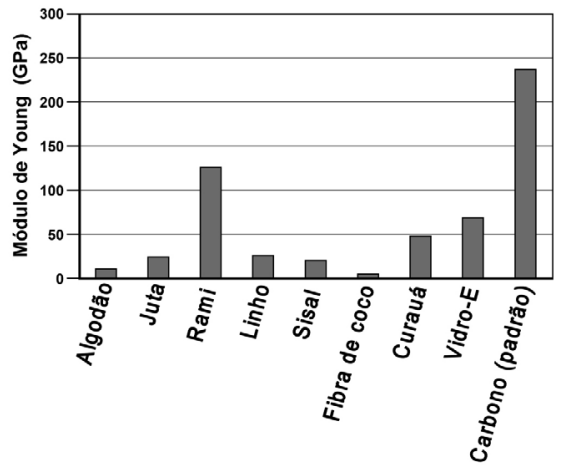

Figura 1. Comparação entre as propriedades mecânicas de fibras naturais e de fibras sintéticas convencionais utilizadas como reforço em compósitos. Adaptada da ref. 10

a)

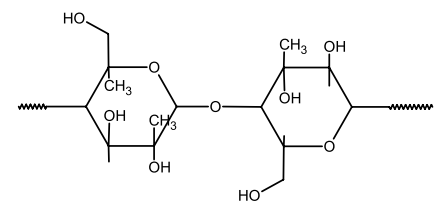

b)

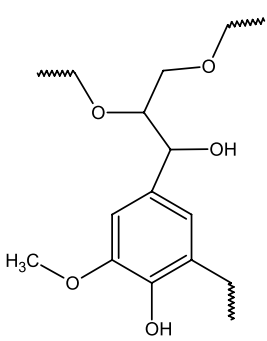

Figura 2. Estrutura química dos componentes das fibras vegetais: a) celulose, b) lignina

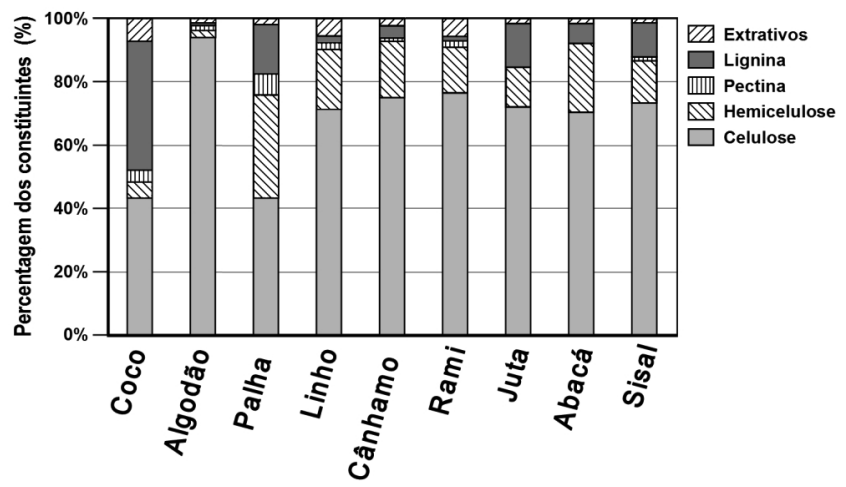

Figura 3. Composição química de algumas fibras vegetais. Adaptada da ref. 18

tipo de substância química que irá reagir com a fibra, permitindo maior interação entre os constituintes do compósito. Essa ligação entre os diferentes materiais pode ser uma ligação química efetiva, especialmente as de caráter covalente, ou do tipo ligação hidrogênio ou, ainda, ácido-base. São listados, a seguir, alguns tipos de tratamentos químicos e sua influência sobre propriedades dos compósitos (fibra + polímero) preparados com a fibra tratada.

\section{Mercerização}

A mercerização, tratamento alcalino, é, provavelmente, o mais popular processo de modificação de fibras naturais. Seu uso pressupõe a solubilização de hemicelulose e de lignina, além de alterar a cristalinidade da celulose. ${ }^{9,11,12} \mathrm{~A}$ hemicelulose é solúvel em baixíssimas concentrações de álcali e, nessas condições, a lignina sofre hidrólise básica (Figura 4). Esse tratamento aumenta a rugosidade da superfície da fibra e melhora a aderência mecânica. ${ }^{20-45} \mathrm{~A}$ mercerização depende da concentração da solução alcalina empregada, da temperatura e do tempo de duração do tratamento. Quando a fibra mercerizada é adicionada a uma matriz polimérica, a adesão ocorre pelo mecanismo

de ancoragem mecânica da fibra, resultando em um maior contato físico entre os dois materiais. ${ }^{21}$<smiles>CCOCC(OCC)C(O)c1cc(CC)c(O)c(OC)c1</smiles><smiles>CCOc1cccc(OCC)c1O</smiles>

Figura 4. Hidrólise básica da lignina

Tratamentos alcalinos, com $\mathrm{KOH}, \mathrm{LiOH}$ e $\mathrm{NaOH},{ }^{20-45}$ levam ao aumento na quantidade de celulose amorfa, devido ao enfraquecimento da ligação hidrogênio na estrutura molecular da celulose, que se apresenta sob a forma de rede. ${ }^{9,11,12} \mathrm{Em}$ presença de água, a estrutura celulósica sofre inchamento, o que acaba por alterar sua cristalinidade (de monoclínica para polimórfica). ${ }^{19} \mathrm{O}$ grau de inchamento e, consequentemente, o tipo de estrutura cristalina obtida dependem do tipo e da concentração da substância alcalina usada no tratamento. ${ }^{23}$

Platenik e colaboradores ${ }^{43}$ estudaram através da microscopia eletrônica de varredura (MEV) como o tempo e a concentração da mercerização modificam a superfície das fibras de bananeira. Foi possível observar que tempos curtos ( 1 e 3 h) de contato do $\mathrm{NaOH}$ com a fibra de bananeira e baixa concentração da base não conseguem modificar a cristalinidade e nem a estrutura da fibra. Existe um tempo e uma concentração ótimos (6 h e 1 molar) de contato para se conseguir retirar a lignina da fibra e deixar a celulose mais exposta, favorecendo a ancoragem mecânica do agente interfacial na fibra (Figura 5).

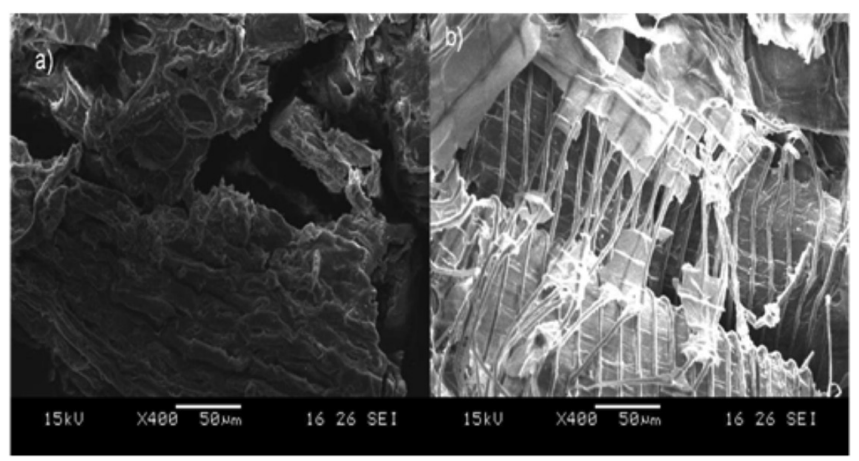

Figura 5. Exemplo da mercerização na fibra de bananeira: a) fibra sem tratamento; b) mercerização (8 h de tratamento a 1 molar). Adaptada da ref. 43 
Cordeiro e colaboradores ${ }^{44}$ caracterizaram as superfícies das fibras de eucalipto cultivado iraniano, abeto, bagaço e palha de trigo, utilizando a técnica de cromatografia gasosa inversa (CGI). Para obter melhores resultados, as fibras foram tratadas com solução $1 \%$ de $\mathrm{NaOH}$ e, após este tratamento, foram misturadas ao polímero para formação do compósito. A cristalinidade das fibras e a interação específica entre a fibra e o polímero foi melhorada com o tratamento alcalino. A CGI mostrou um aumento geral na molhabilidade da fibra modificada, quando comparada com as não modificadas. Os melhores resultados nas propriedades das fibras foram obtidos para as fibras de eucalipto cultivado iraniano, quando comparado com as fibras de madeira.

Arrakhiz e colaboradores ${ }^{45}$ utilizaram dois tipos de tratamentos químicos: o primeiro para tratar as fibras de cone de pinho e o segundo para modificar o polímero. As fibras de cone de pinho foram tratadas com $\mathrm{NaOH}$ para retirar as ceras e os componentes não celulósicos da superfície da fibra. Para garantir a adesão entre a fibra e a matriz polimérica, o polipropileno foi reagido com um copolímero tribloco de estireno-(etileno-buteno)-estireno graftizado com anidrido maleico. As propriedades mecânicas e térmicas do compósito com polipropileno e fibras de cone de pinho foram estudadas por análise termogravimétrica (TGA), testes de tração e de torção.

Arrakhiz e colaboradores ${ }^{45}$ verificaram que a estabilidade térmica depende da quantidade de fibra tratada adicionada ao compósito. Quando se adicionam 8\% em massa de fibra tratada ao compósito, tem-se um aumento na estabilidade térmica; no entanto, ao se aumentar para $25 \%$ em massa de carga ocorre uma elevada redução na estabilidade do compósito. Já as propriedades mecânicas tiveram uma melhoria significativa quando os dois tratamentos químicos foram realizados. Por exemplo, os valores de módulo de Young aumentaram de 43 a $49 \%$.

Huang, ${ }^{25} \mathrm{em}$ seu estudo, aplicou o tratamento alcalino nas fibras secas de coco usando diferentes concentrações de $\mathrm{NaOH}$. Os resultados dessa pesquisa são mostrados nas Figuras 6 e 7. As fibras foram submetidas a esse tratamento por 4 semanas, à temperatura ambiente. Pode-se observar que o aumento da concentração de $\mathrm{NaOH}$ usado no tratamento da fibra provoca uma queda na tensão na ruptura. Mais precisamente, tem-se que a cada $1 \%$ em massa de $\mathrm{NaOH}$ adicionado durante o tratamento da fibra, a tensão na ruptura decresce cerca de $11 \mathrm{cN}\left(10^{-2} \mathrm{~N}\right)$.

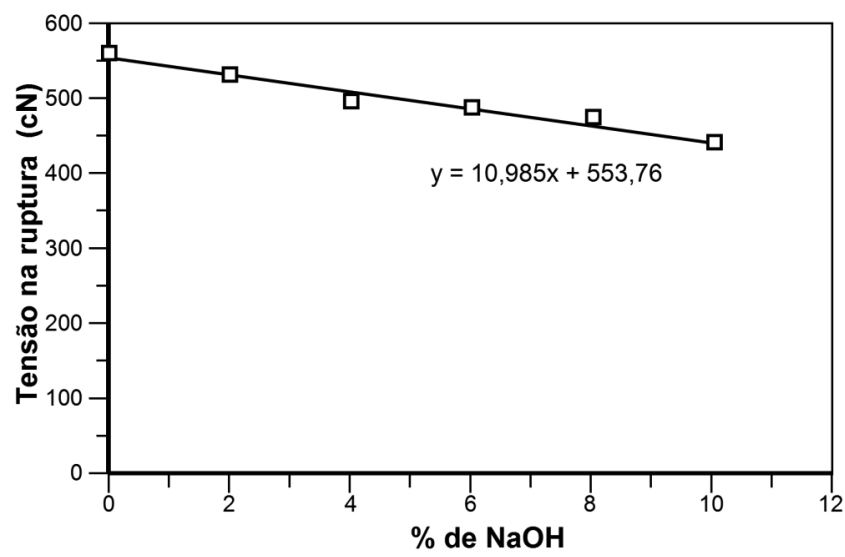

Figura 6. Tensão na ruptura da fibra de coco tratada com soluções de $\mathrm{NaOH}$ em diferentes concentrações. Adaptada da ref. 25

Huang ${ }^{25}$ também observou por microscopia eletrônica de varredura (MEV) que o tratamento alcalino em altas concentrações causa a desestruturação parcial das fibras. $\mathrm{O}$ autor sugere que essa desestruturação leva à diminuição da resistência da fibra, o que se

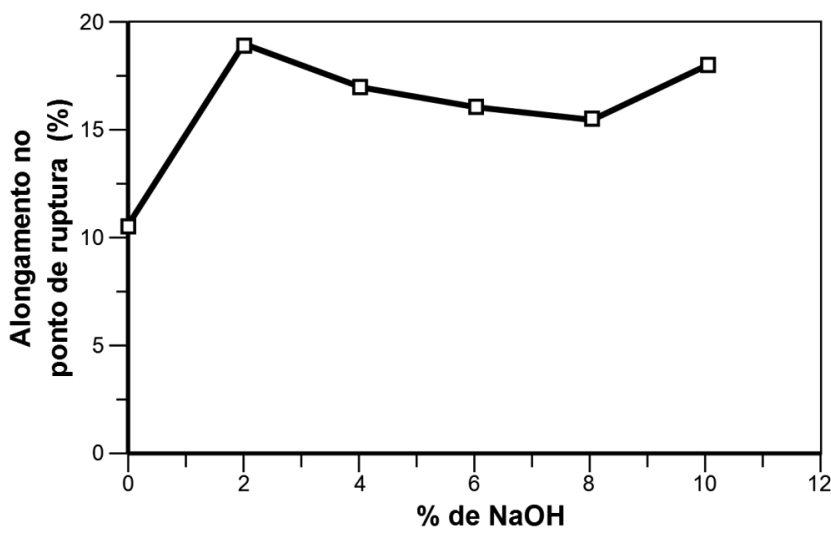

Figura 7. Alongamento no ponto de ruptura da fibra de coco tratada com soluções de $\mathrm{NaOH}$ em diferentes concentrações. Adaptada da ref. 25

explica pela extração da lignina, o principal componente responsável pela adesão entre si das camadas de celulose. A extração de lignina, entre outros compostos, embora acarrete uma redução na resistência da fibra, amplia sua área superficial, devido ao aumento da rugosidade da superfície. Após o tratamento, observa-se um conjunto de orifícios e rugosidades gerados na fibra, que antes do tratamento não eram perceptíveis. A formação de orifícios e rugosidades aumenta a área de contato entre a matriz e a fibra, colaborando para o estabelecimento de mais pontos de interação.

Huang ${ }^{25}$ após verificar que a fibra de coco apresentou uma melhora nas propriedades mecânicas, misturou-a com o polipropileno, formando compósitos de fibra de coco/polipropileno (PP). Esses compósitos apresentaram propriedades mecânicas melhores que o polímero puro, como consequência do aumento da área superficial causado pelo aumento da rugosidade da superfície, o que provoca uma maior interação entre a fibra e o polímero. Na Tabela 1 e Figura 8, encontram-se os resultados apresentados por Huang para o compósito de fibra de coco e polipropileno.

Tabela 1. Propriedades dos compósitos de PP e fibra de coco tratada em diferentes concentrações de $\mathrm{NaOH}$. Adaptada da ref. 25

\begin{tabular}{ccc}
\hline $\begin{array}{c}\text { Teor de NaOH no } \\
\text { tratamento da fibra de } \\
\text { coco (\% massa) }\end{array}$ & $\begin{array}{c}\text { Espessura da fibra } \\
(\mathrm{mm})\end{array}$ & $\begin{array}{c}\text { Alongamento no ponto } \\
\text { de ruptura }(\%)\end{array}$ \\
\hline Fibra original & 1,9 & 15 \\
PP & - & 11 \\
PP/fibra-NaOH 2\%m & 2,0 & 17 \\
PP/fibra-NaOH 4\%m & 2,0 & 17 \\
PP/fibra-NaOH 6\%m & 1,9 & 18 \\
PP/fibra-NaOH 8\%m & 1,9 & 19 \\
PP/fibra-NaOH 10\%m & 2,0 & 19 \\
\hline
\end{tabular}

Observa-se na Figura 8 que todas as concentrações de $\mathrm{NaOH}(2$, 4, 6 e $8 \%$ em massa) utilizadas no tratamento resultam em compósitos com resistência à tração bem maior que o PP puro. Porém, percebe-se que não ocorre uma relação linear entre a concentração de $\mathrm{NaOH}$ e esta propriedade. Pode-se considerar que na faixa de concentrações variando de 2 a $8 \%$ em massa, a resistência à tração do compósito permanece praticamente inalterada, enquanto os compósitos em que se usa a fibra original ou tratada com $\mathrm{NaOH} 10 \%$ em massa apresentam menor resistência ${ }^{25}$

Acetilação e propionilação

A acetilação e a propionilação são métodos de modificação da 


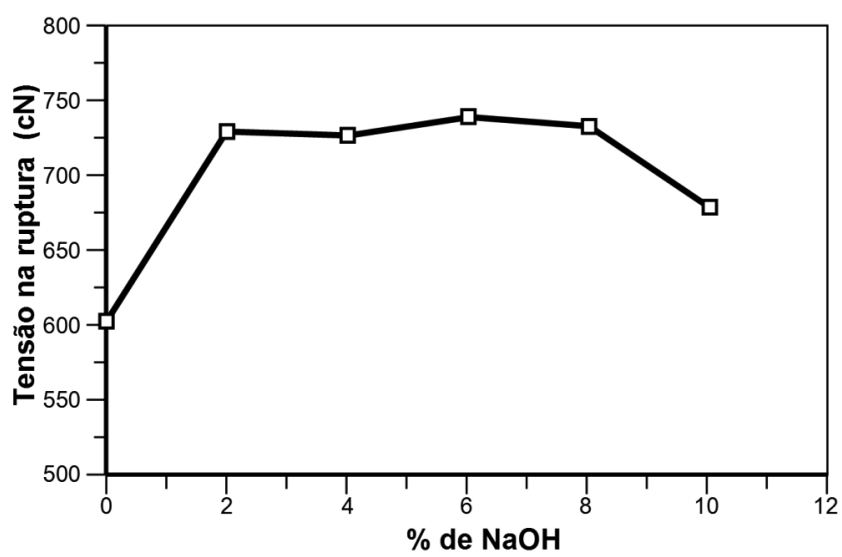

Figura 8. Tensão na ruptura do compósito polipropileno e fibra de coco tratada em diferentes concentrações de $\mathrm{NaOH}$. Adaptada da ref. 22

superfície das fibras que visam torná-las mais hidrofóbicas. .2,20,22,24,46-56 Esse tratamento ocorre pela reação de esterificação do grupo hidroxila dos constituintes da fibra (hemicelulose, lignina e celulose amorfa) com o grupo acetil $\left(\mathrm{CH}_{3} \mathrm{CO}\right)$. A presença de água dificulta essa reação. $\mathrm{O}$ grupo hidroxil dentro das estruturas cristalinas da fibra possui interações muito fortes com a água, que impedem a aproximação do reagente de acetilação. A acetilação do grupo $\mathrm{OH}$ na celulose pode ser vista na Equação $1 .^{22}$

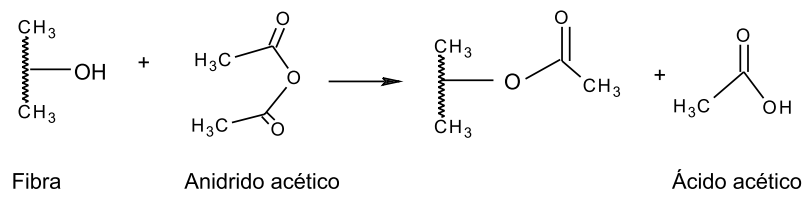

Tserki e colaboradores ${ }^{48}$ trataram as fibras de linho, cânhamo e madeira com anidridos acético e propiônico. Analisaram o grau de esterificação usando o índice de saponificação e espectrometria de absorção na região do infravermelho pelo método de reflectância total atenuada (FTIR-ATR).

O grau de esterificação por grama de fibra, determinado com base no índice de saponificação, é definido como a quantidade em gramas de $\mathrm{KOH}$ necessária para neutralizar os ácidos livres. Os autores observaram que a fibra de madeira apresentou um maior grau de esterificação, devido ao seu alto teor de lignina/hemicelulose. A celulose, embora seja o componente principal de muitas fibras, possui uma estrutura cristalina muito empacotada, o que deixa as hidroxilas da sua estrutura protegidas do ataque de agentes químicos. Por isso, os processos de acetilação e propionilação são mais efetivos em fibras ricas em lignina. Pode-se associar esse aumento da formação do éster à capacidade do anidrido acético de inchar os componentes da fibra, fazendo com que os sítios químicos reativos se tornem mais acessíveis. Após 120 min de reação, não se tem mais um aumento significativo do teor de éster em ambos os tratamentos, indicando um limite de acessibilidade dos sítios, ${ }^{45}$ ou seja, a velocidade de reação está associada à difusão do reagente.

Conclui-se, portanto, que o tipo de material lignocelulósico utilizado durante a esterificação é de extrema importância, já que os três principais componentes da fibra (lignina, hemicelulose e celulose) apresentam reatividades diferentes na presença de anidrido acético ou propiônico. A reatividade do anidrido acético com os constituintes das fibras diminui de acordo com a seguinte sequência: lignina> hemicelulose > celulose.

A espectrometria no infravermelho com transformada de Fourier através do método de reflectância total atenuada (FTIR-ATR) permite analisar a estrutura química dos materiais opacos ou apenas a superfície de um dado material. O FTIR-ATR revelou que a superfície química das fibras foi alterada após os dois tratamentos. Pelos espectros obtidos, conclui-se que o tratamento das fibras de linho e cânhamo com anidridos acético e propiônico levou ao surgimento das bandas de absorção nas regiões $1735-1737 \mathrm{~cm}^{-1}$, referentes à vibração da carbonila de éster, e em 1162-1229 cm cm $^{-1}$ referentes à deformação angular de -O-C-O do éster. Nota-se, nos espectros das três fibras, uma diminuição da banda larga de absorção em $3400 \mathrm{~cm}^{-1}$, referente ao estiramento do hidrogênio da hidroxila. Se a reação fosse completa, ou seja, se todas as hidroxilas da fibra tivessem sofrido esterificação, essa banda não existiria.

O espectro da fibra de madeira tratada é bastante semelhante aos das fibras de linho e de cânhamo, com um pico a mais em $1504 \mathrm{~cm}^{-1}$, associado à vibração do $\mathrm{C}-\mathrm{H}$ do anel aromático, presente principalmente na lignina. ${ }^{48} \mathrm{~A}$ maior proporção de lignina na madeira permite essa diferenciação no espectro.

Lopes e colaboradore ${ }^{20}$ submeteram fibras de sisal ao tratamento de acetilação. Avaliaram a absorção de água, por imersão, e a modificação química das fibras, por FTIR. As fibras de sisal no seu estado natural apresentaram um ganho de massa, por absorção de água, de 81 e $86 \%$, após 2 e 24 h, respectivamente. Quando esses resultados foram comparados aos obtidos para as fibras modificadas por acetilação, observou-se que as fibras acetiladas tiveram um ganho de massa, por absorção, entre 36 e 50\%, em relação à sua massa inicial, quando imersas por $2 \mathrm{~h}$, e entre 39 e $59 \%$, após $24 \mathrm{~h}$, nas temperaturas de 100-120 ${ }^{\circ} \mathrm{C}$ (Tabela 2).

Tabela 2. Absorção de água por fibras de sisal naturais e acetiladas em diferentes tempos e temperaturas de imersão do banho. Adaptada da ref. 20

\begin{tabular}{lccc}
\hline & \multicolumn{3}{c}{ Tempo de imersão } \\
\cline { 2 - 4 } Tipo de tratamento & $30 \mathrm{~min}$ & $2 \mathrm{~h}$ & $24 \mathrm{~h}$ \\
\cline { 2 - 4 } & \multicolumn{3}{c}{ Absorção d água (\% massa) } \\
\hline Natural & 37 & 81 & 86 \\
Acetilada $1 \mathrm{~h} 100{ }^{\circ} \mathrm{C}$ & 18 & 45 & 59 \\
Acetilada $1 \mathrm{~h} 120^{\circ} \mathrm{C}$ & 33 & 36 & 39 \\
Acetilada $2 \mathrm{~h} 110^{\circ} \mathrm{C}$ & 45 & 48 & 47 \\
Acetilada 3 h $100{ }^{\circ} \mathrm{C}$ & 60 & 50 & 51 \\
Acetilada 3 h $120^{\circ} \mathrm{C}$ & &
\end{tabular}

A acetilação das fibras foi verificada por análise de FTIR com o surgimento da banda de carbonila em $1740 \mathrm{~cm}^{-1}$ e pela diminuição da banda da hidroxila em $3400 \mathrm{~cm}^{-1}$, causados pela modificação na composição química das fibras após o tratamento. ${ }^{20}$.

D'Almeida e colaboradores ${ }^{54}$ analisaram o efeito da acetilação sobre a estrutura e a morfologia superficial de fibras de bucha, por meio de FTIR e MEV. Observaram que o tratamento reduziu a polaridade das moléculas de celulose e removeu a camada superficial das fibras de bucha, expondo sua estrutura fibrilar interna, com consequente aumento da área disponível para a adesão.

Ao serem comparados os espectros das fibras natural e acetilada, observa-se, no espectro da fibra natural, a presença de sinal em 3400 $\mathrm{cm}^{-1}$, característico da vibração axial das hidroxilas da celulose, e um sinal em $1690 \mathrm{~cm}^{-1}$, atribuído ao grupo acetal, correspondente à carbonila. No espectro da fibra acetilada, verifica-se uma redução significativa do sinal correspondente à hidroxila. A presença de grupamentos hidroxila na fibra tratada é comprovada pela presença do sinal em $3500 \mathrm{~cm}^{-1}$, referente à vibração axial das hidroxilas, o que indica que a acetilação não foi completa. Por outro lado, o sinal em $3640 \mathrm{~cm}^{-1}$ corresponde ao grupamento $\mathrm{OH}$ da água adsorvida 
Tabela 3. Propriedades mecânicas dos compósitos de polipropileno reforçado com celulose e celulignina acetiladas ou não e dos compostos puros. Adaptada da ref. 55

\begin{tabular}{|c|c|c|c|c|}
\hline $\begin{array}{l}\text { Teor de fibra nas misturas com PP } \\
\text { (\%massa) e identificação da fibra }\end{array}$ & $\begin{array}{l}\text { Tensão na ruptura } \\
(\mathrm{MPa})\end{array}$ & $\begin{array}{l}\text { Alongamento no ponto de } \\
\text { ruptura }(\%)\end{array}$ & $\begin{array}{l}\text { Tensão de flexão } \\
\text { (MPa) }\end{array}$ & $\begin{array}{l}\text { Resistência ao cisalhamento } \\
\text { (MPa) }\end{array}$ \\
\hline $0 \% \mathrm{~m}$ & $27 \pm 1,0$ & $11 \pm 0,2$ & $25 \pm 0,1$ & $7 \pm 0,3$ \\
\hline $10 \% \mathrm{~m}$ de celulose & $26 \pm 0,7$ & $9 \pm 0,4$ & $35 \pm 0,2$ & $10 \pm 0,1$ \\
\hline $20 \% \mathrm{~m}$ de celulose & $26 \pm 0,3$ & $6 \pm 0.3$ & $36 \pm 0,4$ & $11 \pm 0,2$ \\
\hline $10 \% \mathrm{~m}$ de celulose acetilada & $25 \pm 0,8$ & $9 \pm 0,7$ & $28 \pm 0,2$ & $8 \pm 0,2$ \\
\hline $20 \%$ m de celulose acetilada & $20 \pm 0,4$ & $7 \pm 0,4$ & $28 \pm 0,2$ & $8 \pm 0,1$ \\
\hline $10 \% \mathrm{~m}$ de celulignina & $24 \pm 0,5$ & $9 \pm 0,4$ & $32 \pm 0,2$ & $10 \pm 0,3$ \\
\hline $20 \%$ m e de celulignina & $24 \pm 0,3$ & $7 \pm 0,4$ & $34 \pm 0,4$ & $9 \pm 0,2$ \\
\hline $10 \% \mathrm{~m}$ de celulignina acetilada & $23 \pm 0,2$ & $10 \pm 0,2$ & $28 \pm 0,1$ & $9 \pm 0,2$ \\
\hline $20 \%$ m de celulignina acetilada & $20 \pm 0,4$ & $8 \pm 0,3$ & $29 \pm 0,7$ & $12 \pm 0,1$ \\
\hline
\end{tabular}

na fibra. $\mathrm{O}$ sinal intenso em $1722 \mathrm{~cm}^{-1}$ corresponde à carbonila do acetato, confirmando a ocorrência da reação de acetilação da fibra de bucha. Embora a esterificação do grupo $\mathrm{OH}$ não tenha sido total, a reação parcial observada já é potencialmente interessante, pois reduz a higroscopicidade (verificada por análise termogravimétrica-TGA) das fibras, visto que os sítios de hidroxila favorecem a absorção de umidade. $^{54}$

Luz e colaboradores ${ }^{55}$ verificaram as propriedades térmicas por calorimetria exploratória diferencial (DSC) e termogravimentria (TGA) e mecânicas (testes de tração, cisalhamento e flexão) do compósito de polipropileno reforçado com as fibras de celulose e celulignina (fibra sem hemicelulose) do bagaço de cana. O bagaço de cana é constituído por três componentes principais: celulose, hemicelulose e lignina. Nesse trabalho, a celulose e a celulignina foram retiradas, sendo que a celulignina foi obtida a partir da hidrólise do bagaço da cana. Depois disso, a celulignina foi tratada em meio alcalino para remoção de lignina, obtendo-se assim a fibra de celulose. A celulignina é um material novo e promissor que, como a celulose, pode ser usado como reforço em polímeros, devido à sua estrutura fibrosa.

Após o tratamento da fibra, os autores verificaram que o teor de fibras adicionadas aos compósitos de PP com as fibras de celulose e celulignina acetiladas influenciou nas propriedades térmicas e mecânicas do compósito. A caracterização térmica dos compósitos com fibra de celulose e com celulignina acetiladas mostrou uma estabilidade térmica intermediária em relação à matriz e à fibra. Os resultados de DSC revelaram que os compósitos reforçados com fibras não tratadas são mais cristalinos do que o polipropileno puro. ${ }^{55}$

As propriedades mecânicas dos compósitos de PP reforçado com celulose e celulignina, acetiladas e não tratadas (Tabela 3 ) foram analisadas. Os compósitos de PP reforçados com fibras tratadas ou não tratadas apresentaram resistência à tração inferior à do PP puro (27 MPa). Valores inferiores foram também encontrados para o alongamento na ruptura. No entanto, os valores de tensão de flexão e resistência ao cisalhamento mostraram melhora com a introdução da fibra, mas não foi possível observar um efeito positivo do tratamento químico das fibras. ${ }^{55}$

\section{Silanização}

A silanização é um tratamento químico com compostos de silício, cujas moléculas apresentam, em uma das extremidades, um grupo terminal hidrofóbico, que pode desenvolver interação do tipo van der Waals com a matriz do compósito, e, na outra extremidade, um grupo hidrofílico, o qual pode reagir com os grupos $\mathrm{OH}$ da fibra, formando uma ponte. A reação do silano depende de vários fatores, como organofuncionalidade do silano, temperatura e $\mathrm{pH}$. A reação química geral de silanização está apresentada na Figura 9.,9,12,16,22,24,57-61

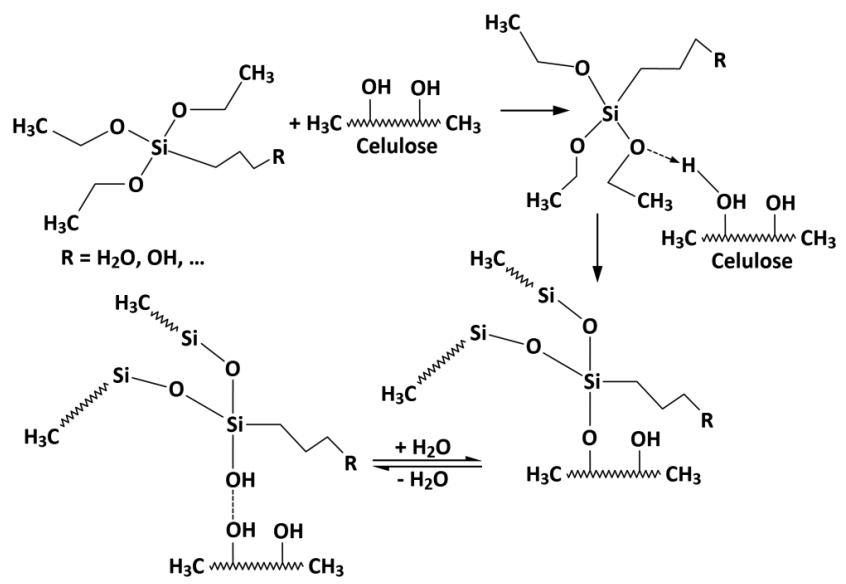

Figura 9. Esquema de interação do grupo silano com a fibra natural. Adaptada da ref. 25

Xie e colaboradores ${ }^{62}$ mostraram como o tratamento químico utilizando silanos (a maioria trialquiloxissilanos) é eficiente na melhoria da interação entre a matriz polimérica olefínica e as fibras naturais e como esse tratamento atua, favorecendo e aumentando as propriedades mecânicas (modulo de Young, tensão na ruptura, etc) dos compósitos.

Bonelli e colaboradores, ${ }^{16}$ com o objetivo de melhorar a adesão entre a fibra de piaçava e o polietileno de alta densidade reciclado (HDPE-r), fizeram o tratamento superficial da fibra com 3-metacriloxipropil-trimetoxi-silano, utilizando dois métodos diferentes: impregnação direta (fibra-ts) e com solução metanólica (fibra-tms). No primeiro, as fibras foram imersas em $100 \mathrm{~mL}$ de solução $5 \%$ de silano em metanol, durante $30 \mathrm{~min}$. A fibra resultante do processo foi denominada de fibra-tms. As fibras foram, em seguida, secas em estufa a $100{ }^{\circ} \mathrm{C}$ por $5 \mathrm{~h}$. No segundo método, as fibras foram impregnadas diretamente por agitação manual com silano na proporção $1 / 3(\mathrm{~m} / \mathrm{m})$ de silano/fibra, sendo chamadas de fibra-ts.

As fibras de piaçava tratadas foram misturadas com polietileno de alta densidade reciclado (HDPE-r), dando origem a compósitos nas proporções 95/5, 90/10 e 85/15\% massa/massa. As misturas foram obtidas em câmara de mistura Haake a $160^{\circ} \mathrm{C}$, por $5 \mathrm{~min}$, com rotação de $60 \mathrm{rpm}$. As propriedades térmicas foram avaliadas por TGA, as mecânicas, por ensaios de flexão e tração, e a morfologia, por MEV. 
Os autores perceberam que a adição de fibra não tratada à matriz polimérica não ocasionou aumento na resistência à flexão, se comparado ao polímero puro, devido à baixa interação interfacial entre o polímero e a fibra. Comportamento semelhante foi observado na incorporação de fibra tratada com solução metanólica de silano, devido a uma provável extração da fração lignânica da fibra pelo metanol. Essa fração lignânica é possivelmente a responsável pelo aumento do reforço estrutural da fibra, assim como pela melhor interação entre ela e o compatibilizante. Já a adição de fibra tratada diretamente com silano, sem solução de metanol, promoveu um aumento na resistência à flexão do compósito, em especial no composto com $15 \%$ em massa de fibra. Os resultados de módulo de flexão confirmam esse comportamento. Os compósitos confeccionados com fibra tratada com silano apresentaram-se mais rígidos e resistentes à flexão (com $5 \%$ de deformação) que os demais, conforme o esperado, uma vez que ocorreu provavelmente um aumento de adesão interfacial entre a fibra e a matriz. ${ }^{16}$

Os autores também investigaram o efeito da percentagem de fibras tratadas e não tratadas na resistência à tração de HDPE reciclado. A adição de fibra não tratada reduziu significativamente a tensão na ruptura, mas não alterou o módulo de elasticidade do HDPE-r. A fragilidade do material é provavelmente explicada pela concentração de tensões na interface entre fibra e matriz. A adição de fibra tratada com solução metanólica de silano aumentou em pequena proporção o módulo de elasticidade do polímero, com redução da tensão na ruptura. Novamente a fibra tratada apenas com silano apresentou os melhores resultados quanto à tensão na ruptura e ao módulo de elasticidade, corroborando o comportamento observado nos ensaios de flexão. ${ }^{16}$

Pickering e colaboradores ${ }^{60}$ utilizaram -aminopropiltrietoxissilano (GS) e diclorodietilsilano (DCS) para a modificação da superfície da fibra de madeira. Adotaram como método a imersão da fibra em solução de silano durante $2 \mathrm{~h}$, com excesso de água, para ocorrência da hidrólise do agente de acoplamento silano. As técnicas de difração de raios-X e espectroscopia de ressonância magnética nuclear (RMN) foram utilizadas para caracterizar as alterações nas fibras de madeira. O RMN comprovou a formação da ligação éter entre a hidroxila da fibra e o DCS, provocada pela reação de silanização. Após a hidrólise (Equação 2), a molécula de silanol reage com o grupo hidroxila da superfície da fibra, formando a ligação éter e o grupo etil, como mostrado na Equação 3, aumentando, assim, a compatibilidade com o polietileno.

$\left(\mathrm{CH}_{3} \mathrm{CH}_{2}\right)_{2} \mathrm{SiCl}_{2}+2 \mathrm{H}_{2} \mathrm{O} \longrightarrow\left(\mathrm{CH}_{3} \mathrm{CH}_{2}\right)_{2} \mathrm{Si}(\mathrm{OH})_{2}+2 \mathrm{HCl}$

diclorodietilsilano

Dietilsilan-2-ol

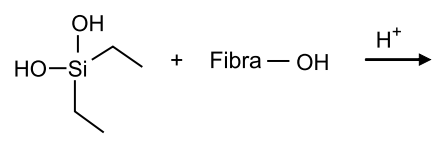

Dietilsilan-2-ol

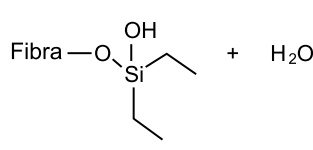

Para o composto GS, a reação ocorre entre o composto de silício e a hidroxila da fibra, após a protonação do grupo amina na presença de ácido acético. O pré-tratamento com $2 \%$ em massa de hidróxido de sódio mostrou-se eficaz na assistência da reação de silanização. O pré-tratamento e o tratamento, entretanto, não contribuíram significativamente para o aumento da resistência mecânica da fibra. ${ }^{60}$

Li e colaboradores ${ }^{61}$ utilizaram dois tipos de tratamentos em fibras de sisal, um de oxidação com permanganato de potássio e dicumilperóxido (DCP) e o outro de silanização. No tratamento por silanização foram usados o 3-aminopropiltrietoxi (silano 1) e o $\gamma$-metacriloxipropiltrimetoxissilano (silano 2). A partir desses tratamentos as fibras foram usadas como reforço no compósito de polietileno de alta densidade. Para descrever a estrutura e as características do compósito, foi utilizado método estatístico. Os valores de resistência ao cisalhamento interfacial (IFSS) das fibras tratadas no compósito HDPE/fibra, obtidos pelo método estatístico, foram menores que os obtidos pelo método convencional. Porém, a IFSS aumentou com os tratamentos de superfície da fibra. Verificou-se que o tratamento com $\mathrm{KMnO}_{4}$ aumenta a IFSS de 1,6 para 3,2 MPa. Com o silano 2, o resultado foi de 2,9 $\mathrm{MPa}$. Sabe-se que $\mathrm{KMnO}_{4} \mathrm{e}$ DCP são oxidantes e podem degradar a superfície da fibra, enquanto o silano é utilizado normalmente como um agente de acoplamento. A modificação química da superfície da fibra com silano 2 gera uma maior interação interfacial com a matriz polimérica por ter uma cadeia principal de carbono mais longa, o que permite o desenvolvimento de interação do tipo van der Waals com a matriz.

\section{MODIFICAÇÃO QUÍMICA DA FIBRA ATRAVÉS DA REAÇÃO COM POLÍMERO OLEFÍNICO MODIFICADO}

Para obter uma compatibilidade entre a matriz polimérica e a fibra, outra possibilidade é modificar quimicamente o polímero, por exemplo, por meio da grafitização com anidrido maleico. ${ }^{4,9,12,13,16,17,24,46,63-80}$ Existem duas possibilidades para a ocorrência de modificação química do polímero. No primeiro caso, tem-se o pré-tratamento do polímero com o produto químico. Durante esse processo, ocorre a reação (geralmente grafitização) do polímero com os compostos que irão aumentar sua polaridade. Após o tratamento, a fibra é adicionada de modo a reagir com o polímero modificado quimicamente. No segundo caso, já se tem o compósito (polímero + fibra), ao qual é adicionado um produto químico que pode reagir com os dois componentes e melhorar a interação entre os materiais. ${ }^{60}$

A grafitização do polímero melhora a molhabilidade da fibra, promovendo uma melhor adesão interfacial, causada pela difusão dos segmentos da cadeia das moléculas grafitizadas na fibra. ${ }^{65} \mathrm{~A}$ eficiência da grafitização é determinada pelo grau de compatibilidade das fibras de celulose com a matriz polimérica. ${ }^{24}$ Alguns parâmetros, como o tipo e a concentração do produto químico e as condições reacionais, afetam a grafitização.

Franco-Marquès e colaboradores ${ }^{78}$ estudaram a influência do uso do polipropileno maleado como agente de acoplamento nos compósitos de polipropileno reforçados com fibras de lignocelulose. Investigaram como a massa molecular do MAH-PP interfere nas propriedades mecânicas dos compósitos, na capacidade de absorção de água e na morfologia da superfície. A utilização do polipropileno maleado funcionalizado com chumbo melhorou as propriedades mecânicas (tensão na ruptura) e também diminuiu a capacidade de absorção de água, devido à formação de interfaces mais finas. $\mathrm{O}$ aumento da massa molecular dos agentes MAH-PP acarreta um aumento na resistência ao impacto, devido a uma menor dispersão do agente de acoplamento na matriz durante o processo de mistura, mantendo microdomínios que ajudam a evitar as rachaduras.

Morandim-Giannetti e colaboradores ${ }^{79}$ estudaram o efeito da incorporação de lignina nos compósitos de polipropileno/fibra de coco na ausência e presença do compatibilizante anidrido maleico nas propriedades térmicas e mecânicas. Os compósitos foram preparados em um reômetro de torque Haake; as propriedades mecânicas foram avaliadas por meio de testes de tração e as propriedades térmicas por análise termogravimétrica (TGA) e calorimetria diferencial exploratória (DSC). Os resultados mostraram que, na ausência de PP-g-MA, a incorporação de lignina não afetou a resistência à tração e, na sua presença, esta propriedade foi reduzida. A análise térmica revelou que a incorporação de lignina nos compósitos resultou no aumento, em ambos os casos, na temperatura inicial de decomposição térmica e nos tempos de indução à oxidação. 
Wong e colaboradores ${ }^{80}$ estudaram o efeito de três agentes de acoplamento de diferentes massas moleculares de polipropileno grafitizado com anidrido maleico nos compósitos reforçados com fibra de carbono reciclada. O potencial de reforço da fibra reciclada foi aumentado através da melhoria da adesão interfacial entre a fibra e a matriz de PP e isto foi feito através da adição de anidrido maleico enxertado no polipropileno. Os efeitos sobre as propriedades mecânicas dos compósitos foram estudados e observou-se que com a adição de MAPP a adesão interfacial foi melhorada, havendo uma melhora significativa na resistência ao cisalhamento, resultando em compósitos com maior resistências à tração e flexão. A força máxima foi alcançada a partir do MAPP com a mais alta massa molecular, obtendo-se um compósito com o maior valor de resistência ao impacto.

Gassan e Bledzki ${ }^{72}$ verificaram a eficácia do copolímero de anidrido maleico com polipropileno, utilizado como compatibilizante nos compósitos de polipropileno/juta. Observaram que o tempo de tratamento da fibra e a concentração de MAH-PP interferem nas propriedades mecânicas do compósito. Houve um aumento da tensão de flexão do compósito com as fibras tratadas com MAH-PP em relação às não tratadas. Esse aumento se tornou mais evidente quando se aumentava o teor adicionado de fibra tratada. $\mathrm{O}$ aumento das propriedades mecânicas foi confirmado por microscopia eletrônica de varredura (MEV), na qual se pode ver uma melhor aderência fibra-matriz.

Outra fibra natural com grande potencial de uso como carga em compósitos poliméricos é a fibra de coco verde.

Amim e Pacheco ${ }^{75}$ estudaram os efeitos causados nas propriedades mecânicas do compósito de polietileno modificado com anidrido maleico e fibra de coco, mostrando que os compostos que tinham MAPE como compatibilizante forneceram melhores resultados de resistência à tração e módulo de elasticidade, devido à melhor interação entre a matriz não polar e a fibra de coco polar.

Leblanc e colaboradores ${ }^{76}$ investigaram os efeitos da fibra de coco verde (GCF) usada como carga em compósitos de polipropileno (PP) e os efeitos do agente compatibilizante anidrido maleico. Foram investigadas as propriedades mecânicas do compósito no estado fundido e no estado sólido, através das técnicas de teste avançado não linear harmônico (estado fundido) e ensaios mecânicos padrão e microscopia eletrônica de varredura (estado sólido). Os compósitos PP-GCF são, assim como os demais, materiais heterogêneos que, no estado fundido, apresentam comportamento viscoelástico não linear, em contraste com o PP puro, que tem uma região viscoelástica linear até a deformação de 50-60\%. O módulo complexo aumenta com a carga de fibra de coco, fornecendo um excelente reforço, aparentemente de origem hidrodinâmica, já que não há qualquer interação química entre a matriz polimérica e as fibras. A adição de polipropileno maleatado melhora a umectação das fibras pelo polímero fundido, mas o efeito é tão pequeno que não se pode afirmar que estejam ocorrendo reações químicas específicas. Dados de módulo de flexão confirmam os efeitos de reforço da fibra e nota-se uma melhoria quando se utiliza o polipropileno maleatado. As microfotografias de SEM mostraram claramente que polipropileno maleatado propicia um melhor molhamento das fibras GC por PP, mas é improvável que ocorram interações químicas entre o polímero e a carga.

Ishizaki e Visconte ${ }^{77}$ estudaram a influência do teor de fibra e das condições de mistura de compósitos de polipropileno e fibras de coco verde, através das caracterizações mecânicas e morfológicas. Os compósitos de polipropileno com 10, 20 e 30\% em volume de fibra de coco verde foram obtidos em câmara de mistura Haake, tendo como variáveis a temperatura de processamento e a velocidade de cisalhamento. O módulo de flexão e as características morfológicas dos diferentes compósitos obtidos foram avaliados e comparados com o polipropileno puro. Observou-se que o melhor desempenho quanto à flexão foi obtido em temperatura de $170{ }^{\circ} \mathrm{C}$, velocidade do rotor de $60 \mathrm{rpm}$ e teor de fibra de coco verde de $30 \%$ em volume. Esses resultados também foram comprovados por análise morfológica.

Além da fibra do coco verde, outros resíduos agrícolas têm sido pesquisados quanto ao seu potencial de aplicação.

Habibi e colaboradores ${ }^{17}$ sugeriram a utilização dos resíduos de bagaço de algodão, arroz, banana e linho, provenientes da agricultura do Egito, para a fabricação de compósitos de polietileno reforçado com as fibras lignocelulósicas. Foram utilizados como compatibilizantes o ácido esteárico e o polietileno grafitizado com anidrido maleico. Avaliaram-se as propriedades mecânicas dos compósitos preparados com LDPE e o polietileno grafitizado com anidrido maleico (MLDPE) e as fibras de cana, banana, talo de algodão e arroz por testes de tensão, verificando-se a influência do tipo e da quantidade da fibra adicionada.

Constatou-se que os compósitos que tiveram como compatibilizante o MLDPE apresentaram valores de módulo de Young mais altos, devido ao maior grau de cristalinidade da estrutura. Esse aumento pode estar associado à reticulação e à adesão covalente da fibra ao anidrido presente na matriz polimérica modificada. A resistência aumenta com o aumento da adição das fibras com MDLPE, e diminui com as misturas que apresentam LDPE não compatibilizadas, sendo este valor próximo ao do polímero puro ( $7 \mathrm{MPa})$. O ponto de ruptura do LDPE é maior que o do MLDPE, provavelmente, devido à menor cristalinidade do primeiro polímero. Compostos mais amorfos possuem estados "borrachosos" à temperatura ambiente e são mais aptos ao alongamento. A adição das fibras diminuiu os valores de resistência à ruptura de forma proporcional à quantidade, independente da natureza da fibra. ${ }^{17}$

As propriedades mecânicas e térmicas do compósito são influenciadas pela forma de adesão entre os compatibilizantes com ácido esteárico e o polietileno de baixa densidade grafitizado com anidrido maleico. Observa-se que os compósitos com MLDPE forneceram melhores propriedades, devido à formação da ligação química entre os grupos anidrido e hidroxila da matriz do polímero e fibra, respectivamente.

\section{CONCLUSÕES}

Os tratamentos químicos (mercerização, silanização e acetilação) utilizados para modificação da estrutura da fibra mostram-se eficientes para melhorar a interação interfacial entre a fibra e a matriz polimérica.

As propriedades mecânicas indicaram que os compósitos preparados a partir de fibra modificada quimicamente podem apresentar melhor desempenho que os preparados com fibras não tratadas. $\mathrm{O}$ grau de melhoria nas propriedades mecânicas do compósito com fibra tratada depende do teor de fibra adicionado ao compósito e da forma de tratamento, o que mostra que existe uma percentagem ótima de fibra para ser inserida no compósito.

Esses resultados da literatura evidenciaram que o uso de compósitos à base de fibras naturais, principalmente aquelas que são resíduos da agricultura, mostra-se promissor do ponto de vista comercial.

O tratamento químico das fibras, portanto, é fundamental para permitir seu uso em compósitos olefínicos. Contudo deve-se atentar para a toxicidade dos compostos químicos utilizados e os impactos ambientais gerados a partir desse tratamento.

\section{AGRADECIMENTOS}

Ao apoio das Empresas Haztec Tecnologia e Planejamento Ambiental S.A., Koleta Ambiental S.A. e Centro de Reciclagem Rio (CRR). Ao apoio concedido por Conselho Nacional de 
Desenvolvimento Científico e Tecnológico (CNPq), Fundação Carlos Chagas Filho de Amparo à Pesquisa do Estado do Rio de Janeiro (FAPERJ) e Coordenação de Aperfeiçoamento de Pessoal de Nível Superior (CAPES) para a realização dessa pesquisa.

\section{REFERÊNCIAS}

1. Suddell, B. C.; Evans, W. J.; International Symposium on Natural Polymers and Composites, São Pedro, Brasil, 2002.

2. Dahlke, B.; Larbig, H.; Scherzer, H. D.; Poltrock R.; J. Cell. Plastics 1998, 34, 361 .

3. Silva, R. V.; Aquino, E. M. F.; Rodrigues, L. P. S.; Barros, A. R. F.; Rev. Matéria 2008, 13, 154.

4. Colom, X.; Carrasco, F.; Pages, P.; Canavate, J.; Compos. Sci. Technol. 2003, 63, 161.

5. Scarpinela, G. D. A.; Dissertação de Mestrado, Universidade de São Paulo, Brasil, 2002.

6. Passos, P. R. A.; Tese de Doutorado, Universidade Federal do Rio de Janeiro, Brasil, 2005.

7. Espert, A.; Vilaplana, F.; Karlsson, S.; Compos. Part A: Appl. Sci. Manuf. 2004, 35, 1267.

8. Joseph, P. V.; Rabello, M. S.; Mattoso, L. H. C.; Joseph, K.; Thomas, S.; Compos. Sci. Technol. 2002, 68, 1357.

9. Ichazo, M. N.; Albano, C.; González, J.; Pereira, R.; Candal, M. V.; Compos. Struct. 2001, 54, 207.

10. Marinelli, A. L.; Monteiro, M. R.; Ambrósio, J. D.; Polímeros: Ciência e Tecnologia 2008, 18, 92.

11. Joseph, K.; Thomas, S.; Pavithran, C.; Polymer 1996, 37, 5139.

12. Li, X.; Tabil, L. G.; Panigrahi, S.; J. Polym. Environ. 2007, 15, 25.

13. Qiu, W.; Endo, T.; Hirotsu, T.; Eur. Polym. J. 2005, 41, 1979.

14. Correa, C. A.; Fonseca, C. N. P.; Neves, S.; Razzino, C. A.; Hage, E. Jr.; Polímeros: Ciência e Tecnologia 2004, 13, 154.

15. Rout, J.; Misra, M.; Tripathy, S. S.; Nayak, S. K.; Mohanty, A. K.; Compos. Sci. Technol. 2003, 61, 1303.

16. Bonelli, C. M. C.; Elzubair, A.; Suarez, J. C. M.; Polímeros: Ciência e Tecnologia 2005, 15, 256.

17. Habibi, Y.; El-Zawawy, W. K.; Ibrahim, M. M.; Dufresne, A.; Compos. Sci. Technol. 2008, 68, 1877.

18. Clemons, C. M.; Caulfield, D. F.; Natural fibers: Functional fillers for plastics, Wiley-VCH Verlag: Weinheim, 2005, chap. 11.

19. Fowler, P. A.; Hughes, J. M.; Elias, R. M.; J. Sci. Food Agric. 2006, 86, 1781.

20. Lopes, F. F. M.; Araújo, G. T.; Nascimento, J. W. B.; Gadelha, T. S.; Rev. Educação Agrícola Superior 2008, 23, 80.

21. Tita, S. P. S.; Paiva, J. M. F.; Frollini, E.; Polímeros: Ciência e Tecnologia 2002, 12, 228.

22. John, M. J.; Anandjiwala, R. D.; Polym. Compos. 2007, 29, 187.

23. Weyenberg, I. V.; Troung, T. C.; Vangrimde, B.; Verpoest, I.; Compos. Part A: Appl. Sci. Manuf. 2006, 37, 1368.

24. George, J.; Sreekala, M. S.; Thomas, S.; Polym. Eng. Sci. 2004, 41, 1471.

25. Huang, G.; Mater. Design 2009, 30, 3931.

26. Cao, Y.; Shibata, S.; Fukumoto, I.; Compos. Part A: Appl. Sci. Manuf. 2006, 37, 423.

27. Das, M.; Chakraborty, D.; J. Appl. Polym. Sci. 2006, 102, 5050.

28. Idicula, M.; Boudenne, A.; Umadevi, L.; Ibos, L.; Candau, Y.; Thomas, S.; Compos. Sci. Technol. 2006, 66, 2719.

29. Samal, R. K.; Panda, B. B.; Rout, S. K.; Mohanty, M.; J. Appl. Polym. Sci. 1995, 58, 745.

30. Rout, J.; Tripaty, S. S.; Nayak, S. K.; Misra, M.; Mohanty, A. K.; J. Appl. Polym. Sci. 2001, 79, 1169.

31. Bledzki, A. K.; Fink, H. P.; Specht, K.; J. Appl. Polym. Sci. 2004, 93, 2150.
32. Aziz, S. H.; Ansell, M. P.; Compos. Sci. Technol. 2004, 64, 1231

33. Pickering, K. L.; Beckermann, G. W.; Alam, S. N.; Foreman, N. J.; Compos. Part A: Appl. Sci. Manuf. 2007, 38, 461.

34. Vilaseca, F.; Mendez, J. A.; Pelach, A.; Liop, M.; Cañigueral, N.; Gironès, J.; Turon, X.; Mutjè, P.; Process Biochem. 2007, 42, 329.

35. Ray, D.; Sarkar, B. K.; Rana, A. K.; J. Appl. Polym. Sci. 2002, 85, 2588.

36. Edeerozey, A. M. M.; Ahil, H. M.; Azhar, A. B.; Ariffin, M. I. Z.; Mater. Lett. 2007, 61, 2023.

37. Joseph, S.; Joseph, K.; Thomas, S.; Int. J. Polym. Mater. 2006, 55, 925.

38. Goda, K.; Sreekala, M. S.; Gomes, A.; Kaji, T.; Ohji, J.; Compos. Part A: Appl. Sci. Manuf. 2006, 37, 2213.

39. Alvarez, V. A.; Vazquez, A.; Compos. Part A: Appl. Sci. Manuf. 2006, 37, 1672 .

40. Jacob, M.; Francis, B.; Thomas, S.; Varuguese, K. T.; Polym. Compos. 2006, 27, 671 .

41. Martins, M. A.; Forato, L. A.; Mattoso, L. H. C.; Colnago, L. A.; Carbohydr. Polym. 2006, 64, 127.

42. Shibata, M.; Ozawa, K.; Teramoto, N.; Yosomiya, R.; Takeishi, H.; Macromol. Mater. Eng. 2003, 288, 35.

43. Platenik, G.; Albinante, S. R.; Pacheco, E. B. A. V.; Visconte, L. L. Y.; XXXIII Jornada Giulio Massarani de Iniciação Científica, Artística e Cultural, Rio de Janeiro, Brasil, 2012.

44. Cordeiro, N.; Ornelas, M.; Ashori, A.; Sheshmani, S.; Norouzi, H.; Carbohydr. Polym. 2012, 87, 2367.

45. Arrakhiz, F. Z.; El Achaby, M.; Benmoussa, K.; Bouhfid, R.; Essassi, E.; Qaiss, A.; Mater. Desig. 2012, 40, 528.

46. Tserki, V.; Matzinos, P.; Zafeiropoulos, N. E.; Panayiotou, C.; J. Appl. Polym. Sci. 2006, 100, 4703.

47. Zafeiropoulus, N. E.; Dijon, G. G.; Baillie, C. A.; Compos. Part A: Appl. Sci. Manuf. 2007, 38, 621.

48. Tserki, V.; Zafeiropoulus, N. E.; Simon, F.; Panayiotou, C.; Compos. Part A: Appl. Sci. Manuf. 2005, 36, 1110.

49. Zafeiropoulos, N. E.; Williams, D. R.; Baille, C. A.; Matthews, F. L.; Compos. Part A: Appl. Sci. Manuf. 2002, 33, 1083.

50. Hill, C. A. S.; Khalil, A. H. P. S.; Hale, M. D.; Ind. Crops Products 1998 , 8,53 .

51. Khalil, H. P. S. A.; Ismail, H.; Rozman, H. D.; Ahmad, M. N.; Eur. Polym. J. 2001, 37, 1037.

52. Menezes, A. J.; Pasquini, D.; Curvelo, A. A. S.; Gandini, A.; Carbohyd. Polym. 2009, 76, 437.

53. Mohanty, A. K.; Misra, M.; Drzal, L. T.; Compos. Interfaces 2001, 8, 313.

54. D’Almeida, A. L. F. S.; Calado, V.; Barreto, D. W.; Polímeros: Ciência e Tecnologia 2005, 15, 59.

55. Luz, S. M.; Tio, J.; Rocha, G. J. M.; Gonçalves, A. R.; Del' Arco, A. P.; Compos. Part A: Appl. Sci. Manuf. 2008, 39, 1362.

56. Taib, R. M.; Ramard, S.; Ishak, Z. A. M.; Rozman, H. D.; J. Reinforced Plastics Compos. 2010, 29, 431.

57. Jacob, M.; Francis, B.; Thomas, S.; Varuguese, K. T.; Macrom. Mat. Eng. 2006, 291, 1119.

58. Herrera-Franco, P. J.; Valadez-González, A.; Compos. Part B: Eng. 2005, 36, 597.

59. Demir, H.; Atikler, U.; Balköse, D.; Tihminlioglu, F.; Compos. Part A: Appl. Sci. Manuf. 2006, 37, 457.

60. Pickering, K. L.; Abdalla, A.; Ji, C.; Mcdonald, A. G.; Franich, R. A.; Compos. Part A: Appl. Sci. Manuf. 2003, 34, 915.

61. Li, Y.; Hu, C.; Yu, Y.; Compos. Part A: Appl. Sci. Manuf. 2008, 39, 570.

62. Xie, Y. ; Hill, C. A. S.; Xiao, Z.; Militz, H.; Mai, C.; Compos. Part A: Appl. Sci. Manuf. 2010, 41, 806.

63. Machado, A. V.; Covas, J. A.; Duin, M. V.; Polymer 2001, 42, 3649.

64. Keener, T. J.; Stuart, R. K.; Brown, T. K.; Compos.: Part A: Appl. Sci. Manuf. 2004, 35, 357.

65. Brahmakumar, M.; Pavithram, C.; Pillai, R. M.; Compos. Sci. Technol. 2005, 65, 563. 
66. Lei, Y.; Wu, Q.; Yao, F.; Xu, Y.; Compos.: Part A: Appl. Sci. Manuf. 2007, 38, 1664.

67. Bengtsson, M.; Baillif, M. L.; Oksman, K.; Compos.: Part A: Appl. Sci. Manuf. 2007, 38, 1922.

68. Kaddami, H.; Dufresne, A.; Khelifi, B.; Bendhaou, A.; Taourirte, M.; Raihane, M.; Issartel, N.; Sautereau, H.; Gérard, J. F.; San, N.; Compos.: Part A: Appl. Sci. Manuf. 2006, 37, 1413.

69. Chuai, C. H.; Almdal, K.; Poulsen, L.; Plackett, D.; J. Appl. Polym. Sci. 2001, 80, 2833.

70. Machado, M. A. L.; Arroyo, M.; Biagiotti, J.; Kenny, J. M.; J. Appl. Polym. Sci. 2003, 90, 2170.

71. Joly, C.; Gauthier, R.; Escoubes, M.; J. Appl. Polym. Sci. 1996, 61, 57.

72. Gassan, J.; Bledzki, A. K.; Compos.: Part A: Appl. Sci. Manuf. 1997, 28, 1001 .

73. Nourbakhsh, A.; Ashori, A.; J. Compos. Mater. 2009, 43, 877.
74. Mengeloglu, F.; Karakus, K.; Sensors 2008, 8, 500.

75. Amin, P. R. P.; Dissertação de Mestrado, Universidade Federal do Rio de Janeiro, Brasil, 2006.

76. Leblanc, J. L.; Furtado, C. R. G.; Leite, M. C. A. M.; Visconte, L. L. Y; Ishizaki, M. H.; J. Appl. Polym. Sci. 2006, 102, 1922.

77. Ishizaki, M. H.; Visconte, L. L. Y.; Furtado, C. R. G.; Leite, M. C. A. M.; Leblanc, J. L.; Polímeros: Ciência e Tecnologia 2006, 16, 182.

78. Franco-Marquès, E.; Méndez, J. A.; Pèlach, M. A.; Vilaseca, F.; Bayer, J.; Mutjé, P.; Chem. Eng. J. 2011, 166, 1170.

79. Morandim-Giannetti, A. A.; Agnelli, J. A. M.; Lanças, B. Z.; Magnabosco, R.; Casarin, S. A.; Bettini, S. H. P.; Carbohyd. Polym. 2012, 87, 2563.

80. Wong, K. H.; Mohammed, D. S.; Pickering, S. J.; Brooks, R.; Compos. Sci. Technol. 2012, 72, 835. 